\title{
Functional Group-Selective Ion-Molecule Reactions of Ethylene Glycol and Its Monomethyl and Dimethyl Ethers
}

\author{
Erika S. Eichmann and Jennifer S. Brodbelt \\ Department of Chemistry and Biochemistry, University of Texas, Austin, Texas, USA
}

\begin{abstract}
The selective methylation and methylene substitution reactions of dimethyl ether ions with ethylene glycol, ethylene glycol monomethyl ether, and ethylene glycol dimethyl ether were investigated in a quadrupole ion trap mass spectrometer. Whereas the reactions of ethylene glycol and ethylene glycol monomethyl ether with the methoxymethylene cation $45^{+}$gave only $[\mathrm{M}+13]^{+}$product ions, the reaction of ethylene glycol dimethyl ether with the same reagent ion yielded exclusively $[\mathrm{M}+15]^{+}$ions. The relative rates of formation of these products and those from competing reactions were examined and rationalized on the basis of structural and electronic considerations. The heats of formation for various relevant species were estimated by computational methods and showed that the reactions leading to the $[\mathrm{M}+13]^{+}$ions were more energetically favorable than those leading to the $[\mathrm{M}+15]^{+}$ products for cases in which both reactions are possible. Finally, the collision-induced dissociation behavior of the $[\mathrm{M}+\mathrm{H}]^{+},[\mathrm{M}+13]^{+}$, and $[\mathrm{M}+15]^{+}$ions indicated that the $[\mathrm{M}+\mathrm{H}]^{+}$and $[\mathrm{M}+15]^{+}$ions dissociated by analogous pathways and were thus structurally similar, whereas the $[\mathrm{M}+13]^{+}$ions possessed distinctly different structural characteristics. (f Am Soc Mass Spectrom 1993, 4, 97-105)
\end{abstract}

$\mathrm{T}$ The importance of functional group interactions and substituent effects in determining the outcomes of reactions both in solution [1-4] and in the gas phase has long been recognized [5-8]. For example, the interactions between various functional groups in diol [9], diacid [10], diester [11], and other simple systems [12-14] have been shown to have important consequences on the physical properties and reactive and dissociative patterns of these ions. In some cases, remote group participation can promote reactive channels inaccessible to related molecules lacking the interaction [15]. The converse is also true: The presence of an additional functional group can prevent certain reactions, either through steric or electronic interactions or by promotion of competition between reactions that would otherwise be expected to predominate.

The type and extent of interaction and consequent enhancement or inhibition of the reactions naturally depends on the nature, position, and orientation of all functional groups involved. The large body of previous work in this area has helped to establish generally accepted correlations of functional group interactions with gas-phase basicities and proton affinities, ion sta-

Address reprint requests to Jeruifer S. Brodbelt, Department of Chemistry and Biochemistry, University of Texas, Austin, TX 78712. bilities, associative properties, and the favorabilities of competitive dissociative channels of various types of ions with a variety of functional groups [1-14, 16, 17], An understanding of such functional group interactions is important not only from a physical organic perspective in predicting reaction outcomes and mechanisms but also from a biological standpoint. For example, hydroxyl groups and ether linkages are among the most ubiquitous functional groups [18], and virtually all possible combinations, relative positions, and orientations of these functionalities can be found in sugars, steroids, antibiotics, and other biologically relevant molecules.

Mass spectrometric methods have been increasingly applied to the characterization of such types of biomolecules; however, the structural elucidation of these complex molecules remains deficient. The development of activation techniques, such as collisioninduced dissociation [19] and surface-induced dissociation [20], for promoting fragmentations of biomolecules in characteristic patterns has assisted in solving this problem. The design of selective ion-molecule reactions also holds promise for revealing structurally diagnostic information. Chemical ionization $[21,22]$ reactions with novel reagent gases have been shown to offer great potential, and tremendous interest has therefore been stimulated in the characterization of new site-selective reagents [23]. 
We are particularly concerned with the development of site-specific reactions for characterization of antibiotics, and a firm understanding of the fundamental reactions between the common substituents is therefore a necessity. We have undertaken this study of simple disubstituted ethanes to illustrate that the reactive and dissociative properties of structurally and electronically similar ions can be dramatically different. To correlate and contrast the reactivities of methoxyl and hydroxyl groups, we have compared the ion-molecule reactions of dimethyl ether ions with ethylene glycol and its mono- and diethers and examined the formation mechanisms for each product observed. In addition, we have made a qualitative comparison of the formation rates and relative favorabilities of formation for the observed products and investigated the thermodynamic properties that presumably govern the reactions observed.

The two reactions of interest in this study are methylene substitution and methyl cation attachment. The former has been recently described for other small organic systems $[9,12,24]$. The methyl cation attachment process has also lately been of interest in studies concerning sites of electrophilic additions [25-27].

\section{Experimental}

A Finnigan ion trap mass spectrometer (Finnigan-MAT, San Jose, CA) [28, 29] was used for all experiments. The samples (Aldrich Chemical Co., Milwaukee, WI) were introduced through a heated leak valve system, and typical pressures used were $1.3 \times 10^{-4} \mathrm{~Pa}$. Dimethyl ether (DME) (MG Industries, Valley Forge, PA) reagent gas pressure was generally $1.2 \times 10^{-3} \mathrm{~Pa}$, and helium buffer gas was admitted at approximately $0.13 \mathrm{~Pa}$. The ions produced by electron ionization of DME were stored and reacted with the neutral sample vapor. The ion-molecule reaction times were varied between 0 and $500 \mathrm{~ms}$. Alternately, individual reagent gas ions were trapped and isolated by application of appropriate radiofrequency and dc voltages [30] and allowed to react with the neutral analyte molecules for varying periods of time $(0-500 \mathrm{~ms})$. In either case, the ions formed were selectively isolated, and activated to produce collision-induced dissociation (CID) spectra.

All thermochemical values not available in the literature were estimated by computer calculations. The computational programs PCMODEL and MOPAC were obtained from Serena Software (Bloomington, IN) and were run on a Macintosh IIsi personal computer. The molecular modeling program PCMODEL was first used to approximate the minimum-energy structure, and the resulting coordinates were entered into the semiempirical program MOPAC. The AM1 Hamiltonian operator and default parameters were used in all cases. Calculations were performed at least three times, and consistent values were obtained.

\section{Results and Discussion}

\section{Comparison of Reactions}

Two reactive ions are typically formed on ionization of DMF-the methoxymethylene cation (1) and the protonated DME (2). Previous studies in our group $[24,31]$ have shown that on reaction with the ions of $m / z 45$ and 47 from DME, substrates typically yield one or more of several product ions, depending on the nature, position, and orientation of their functional groups: $[\mathrm{M}+1]^{+},[\mathrm{M}+13]^{+},[\mathrm{M}+15]^{+},[\mathrm{M}+45]^{+}$, and $[\mathrm{M}$ $+47]^{+}$. Although the $[M+45]^{+}$and $[M+47]^{+}$ adduct ions, or collision complexes, are not always observable in the ion trap, previous work has demonstrated that the $[\mathrm{M}+1]^{+},[\mathrm{M}+13]^{+}$, and $[\mathrm{M}+15]^{+}$ ions all originate directly from these ions $[12,31]$.<smiles>C=[O+]C</smiles><smiles>C[OH+]C</smiles>

$45^{+}$

$47^{+}$

1

2

The $[\mathrm{M}+1]^{+}$ions arise predominantly from simple proton transfer from the protonated DME molecule $(\mathrm{m} / z 47)$ to the substrate. These products presumably arise through initial formation of a proton-bound collision complex at $[\mathrm{M}+47]^{+}$, which fragments to give the protonated analyte (Scheme Ia). In most of the systems studied to date in our laboratory, including the three systems under study here, the proton affinities of the substrates have exceeded that of the neutral DME. Therefore, dissociation of the lonsely bound [M $+47]^{+}$collision complex generally gives preferentially the $[\mathrm{M}+1]^{+}$product ion.

Likewise, the $[\mathrm{M}+13]^{+}$and $[\mathrm{M}+15]^{+}$ions have been shown to result from fragmentation of an $[\mathrm{M}+$ $45]^{+}$adduct that arises from the collision complex formed between the neutral analyte and the DME reagent ion at $m / z$ 45 [12]. The complex either rearranges to allow transfer of a methyl group to the substrate (Scheme $\mathrm{lb}$, upper path) and simultaneous loss of formaldehyde or undergoes a different rearrangement followed by loss of methanol (Scheme $\mathrm{Ib}$, lower path), resulting in a net substitution of a methylene group onto the substrate.

For ethylene glycol, ethylene glycol monomethyl ether, and ethylene glycol DME, the $[\mathrm{M}+45]^{+}$and $[\mathrm{M}+47]^{+}$ions are not directly observable, presumably because they are formed with excessive internal energy and are not sufficiently deactivated by collisions with the helium buffer gas. Rather, they dissociate spontaneously on formation, giving $[\mathrm{M}+1]^{+},[\mathrm{M}$ $+13]^{+}$, and $[M+15]^{+}$product ions. 


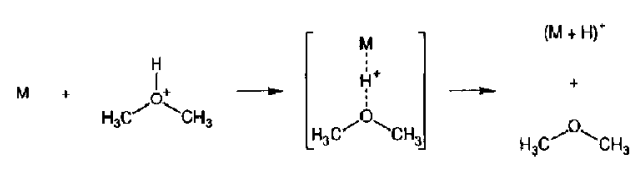

$$
\begin{aligned}
& \begin{array}{cc}
47^{+} & (M+47)^{+} \\
\text {Collision Complex }
\end{array}
\end{aligned}
$$$$
\text { a }
$$

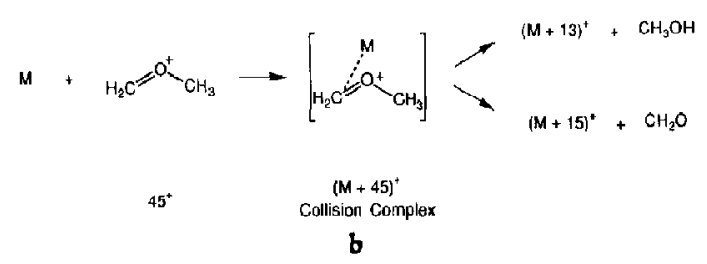

Scheme I. Formation reactions for $(a)[M+1]^{+}$and (b) $[M+$ $13]^{+}$and $[M+15]^{+}$product ions.

Whereas all of these compounds form the protonated molecule $[M+1]^{+}$, the reactions leading to $[M$ $+13]^{\prime}$ and $[M+15]^{+}$are more selective. Ethylene glycol exclusively forms $[M+13]^{+}$ions, whereas the diether only gives $[\mathrm{M}+15]^{+}$ions and no $[\mathrm{M}+13]^{+}$ product. Most interesting, the monoether yields only $[\mathrm{M}+13]^{+}$product ions, even though the formation of $[\mathrm{M}+15]^{+}$ions would also be predicted on the basis of the behavior of the ethylene glycol DME. The fact that a mixture of $[\mathrm{M}+13]^{+}$and $[\mathrm{M}+15]^{+}$products is not observed for this substrate, which contains both of the necessary reactive groups, is especially intriguing. This suggests that the two types of reactions are noncompetitive and that the reagent ion that results in their formation is not only selective, but also gives sitespecific reactions. The nature of both functional groups within the difunctional molecule thus has a significant effect on the reactivity of the compound.

\section{Structural Characterization by Collision-Induced Dissociation}

To evaluate the structures of these product ions, their CID spectra were examined (Figure 1). In each case, only one fragment ion was formed. Collisional activation resulted in loss of neutral formaldehyde for the $[\mathrm{M}+13]^{+}$products and loss of neutral DME from the $[\mathrm{M}+15]^{+}$product.

A comparison of the CID spectra of the $[M+1]^{+}$ and $[\mathrm{M}+15]^{+}$ions for ethylene glycol DME (Figure 2) shows that the protonated and methylated molecules follow analogous dissociation paths. Whereas the $[\mathrm{M}$ $+15]^{+}$product shows exclusive loss of neutral DME, the protonated molecule exclusively loses a molecule of methanol on activation. In both cases, the same product ion at $m / z 59$ is formed. In contrast, the $[\mathbf{M}+1]^{+}$and $[M+13]^{+}$products for ethylene glycol or ethylene glycol monomethyl ether do not dissociate

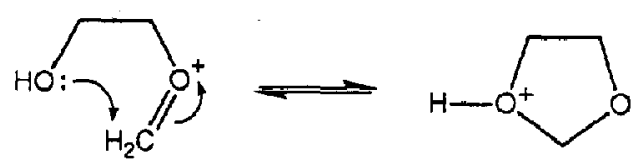

$101 \mathrm{kcal} / \mathrm{mol}$

$99 \mathrm{kcal} / \mathrm{mol}$

a
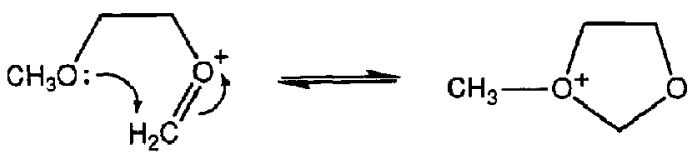

$99 \mathrm{kcal} / \mathrm{mol}$

$94 \mathrm{kcal} / \mathrm{mol}$

b

Figure 1. Estimated heats of formation for the cyclized and uncyclized $[\mathrm{M}+13]^{+}$ions of (a) ethylene glycol and (b) ethylene glycol monomethyl ether.

by parallel paths, supporting the proposal that the protonation and methylene substitution reactions do not result in similar structure types.

We have previously established that whereas the $[\mathbf{M}+13]^{+}$ions of amino alcohols [12] exist predominantly in the uncyclized form, cyclization of the same products derived from diols [9] is significantly favored. In the case of ethylene glycol and ethylene glycol monomethyl ether, the estimated energy differ-. ence between the open and cyclized forms of the ions is relatively small (Figure 3 ).

In both cases, the cyclic form is slightly preferred. This is in accord with deprotonation experiments with the ethylene glycol system described later that demonstrate that a significant amount of the ion abundance exists as the cyclized ion. The fact that neutral DME easily deprotonates the $[M+13]^{+}$product ions is best explained by the presence of the cyclic ions, in which there is an acidic proton. The cyclic $[\mathrm{M}+13]^{+}$ion of ethylene glycol monomethyl ether does not possess an acidic proton, and, consequently, the deprotonation reaction does not occur.

Un the other hand, the simple fragmentations of the $[\mathrm{M}+13]^{+}$ions of both ethylene glycol and its methyl ether on collisional activation suggest that the cyclic structures open on activation, prior to fragmentation. The simple loss of neutral formaldehyde seen exclusively in both cases is most easily explained by direct cleavage of the carbon to positively charged oxygen atom bond in the acyclic $[\mathrm{M}+13]^{+}$ion. The relatively small energy difference between the cyclic and acyclic forms is in accord with the assumption that they are readily interconvertible after collisional activation.

\section{Reaction Profiles}

One of our initial purposes in this work was to investigate the reaction profiles of the observed reactions. Log 


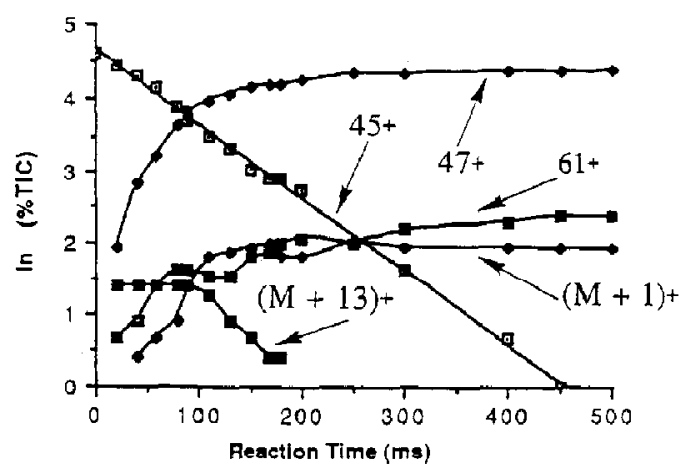

a

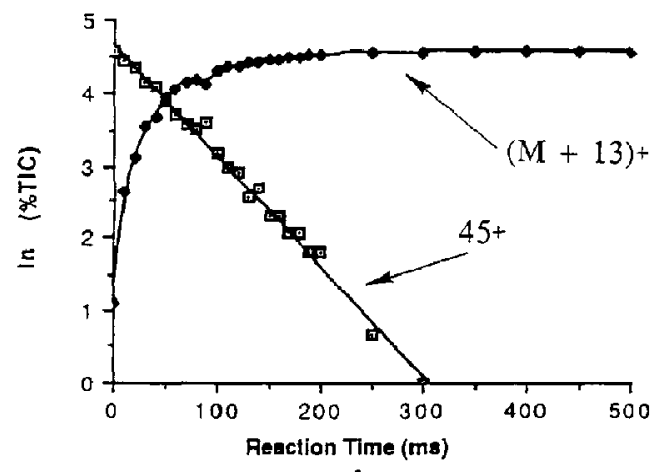

b

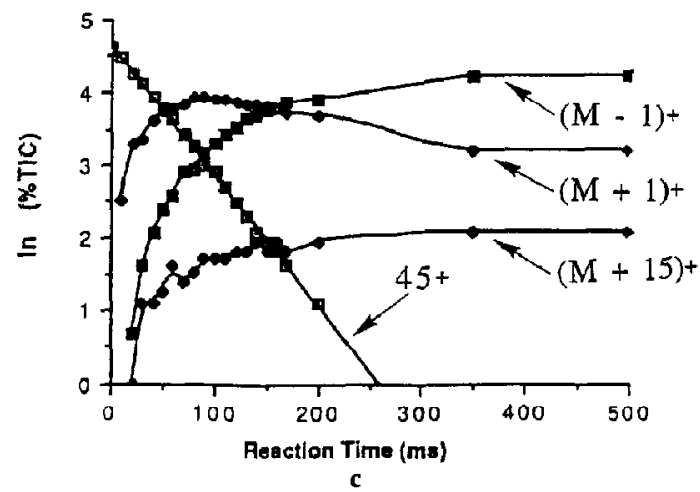

Figure 2. Profile data for the reactions of (a) ethylene glycol; (b) ethylene glycol monomethyl ether; and (c) ethylene glycol DME with the $45^{+}$ion.

plots of the profile data for the ion-molecule reactions of each substrate are shown in Figure 2. For each plot, the DME methoxymethylene $45^{+}$ion was isolated and allowed to react for $0-500 \mathrm{~ms}$ with the analyte. Because of the abundance of products in two of the reactions, little quantitative information can be obtained from these plots; however, several interesting points can be made.

Reaction of ethylene glycol. For the reaction of ethylene glycol and the $45^{+}$cation, many different products are seen (Figure 4a). The fact that the natural log of the
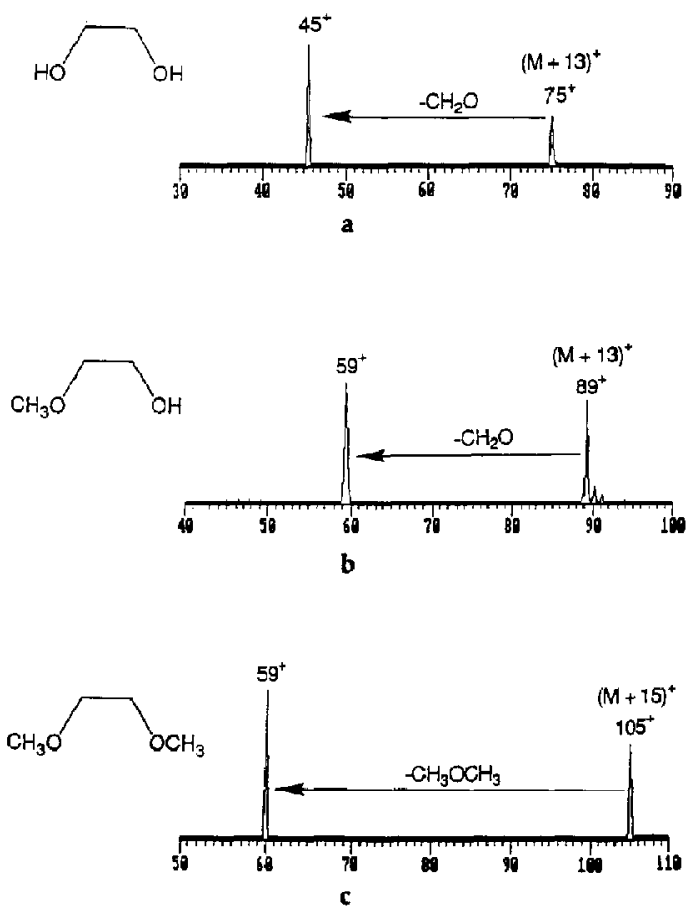

Figure 3. Comparison of the CID spectra of the $[M+13]^{+}$ products of (a) ethylene glycol and (b) ethylene glycol monomethyl ether and the $[\mathrm{M}+15]^{+}$product of $(c)$ ethylene glycol DME.
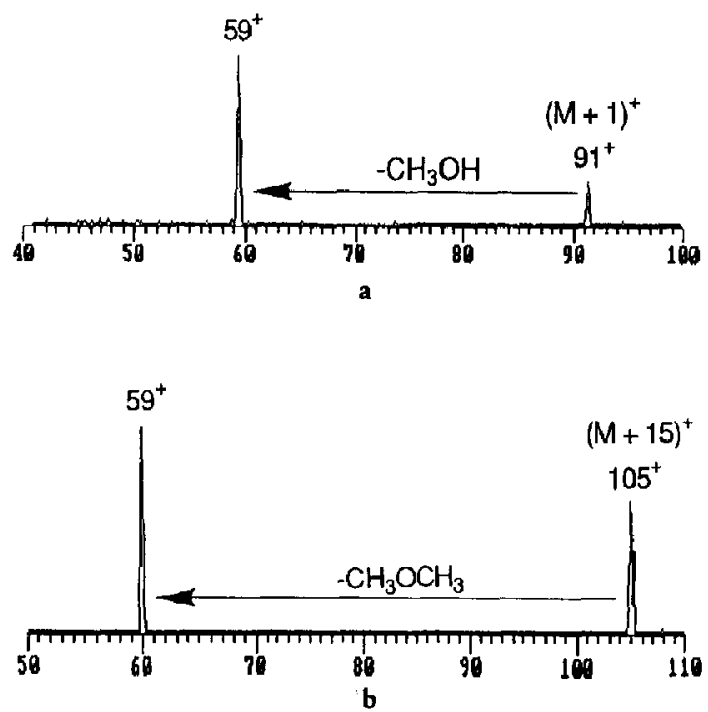

Figure 4. Comparison of the CID behavior of (a) protonated ethylene glycol DME and (b) the $[\mathrm{M}+15]^{+}$product of ethylene glycol DME. 
$45^{+}$ion intensity falls off at a constant rate indicates that most of the products seen are secondary, formed after initial reaction of the substrate with the reagent ion. In fact, the immediate appearance of the $47^{+}$ion is presumably a result of deprotonation of the newly formed $[\mathrm{M}+13]^{+}$ion by neutral DME. This observation would also account for the lowered intensity of $[\mathrm{M}+13]^{+}$at longer reaction times. After the $[\mathrm{M}+$ $13]^{+}$ion current is depleted, the $47^{+}$intensity ceases to rise. This proton transfer process is further confirmed by isolation and storage of the $[\mathrm{M}+13]^{+}$ion. The disappearance of $[\mathrm{M}+13]^{+}$and the concurrent appearance and rise of the $47^{+}$signal indicates that DME does in fact remove a proton from the product ion after it is formed. Again, this idea is confirmed by the fact that the proton affinity of DME is $14 \mathrm{kcal} / \mathrm{mol}$ higher than that of the conjugate base of the proposed $[M+13]^{+}$ion structure.

The appearance of $[\mathrm{M}+1]^{+}$ions during the reaction is thus probably a result of the formation of the $47^{+}$ion. This conclusion is substantiated by examination of the proton affinity of the ethylene glycol. The proton affinity of DME is in this case lower by 14 $\mathrm{kcal} / \mathrm{mol}$ than that of neutral ethylene glycol.

Two possible explanations are offered for the appearance of the $m / z 61$ ions in the course of the reaction of ethylene glycol with the $45^{+}$cation. One source of these ions could be a hydride transfer reaction between ethylene glycol and the $45^{+}$ion that competes with the desired reaction and results in formation of $[M-1]^{+}$ions. Although this reaction is estimated to be enduthermic by $3 \mathrm{kcal} / \mathrm{mol}$ overall, the internal temperature of the ions in the trap may cause it to occur nonetheless. Another possibility is that the $61^{+}$signal is not the $[\mathrm{M}-1]^{+}$ion of ethylene glycol, but is instead due to a methyl cation transfer reaction between the $45^{+}$cation and the neutral DME present in the system. This process is commonly observed at long reaction times in many cases. The high relative abundance of this ion in the reaction profile of ethylene glycol, along with the lack of this ion in the profiles of the other two substrates (Figure $2 b$ and $c$ ), suggests that the hydride abstraction reaction is the most likely explanation for this ion.

Reaction of ethylene glycol monomethyl ether. In contrast to the ethylene glycol reaction described earlier, only the product of interest in this study, $[M+13]^{+}$, is formed from the reaction of ethylene glycol monomethyl ether with the $45^{+}$ion (Figure $2 \mathrm{~b}$ ). Because the $[M+13]^{+}$signal does not rise at the same rate as the $45^{+}$signal falls, it can be inferred that the conversion efficiency is not $100 \%$. Processes that may account for the moderate conversion efficiency include ion leakage from the trap and neutralization of the $45^{+}$ ions; however, the relative intensity of the $[\mathrm{M}+13]^{+}$ product as compared with that observed in the case of ethylene glycol is large, and no detectable side products are formed. Proton transfer to neutral DME from the $[\mathrm{M}+13]^{+}$ion and hydride abstraction from neutral ethylene glycol monomethyl ether by the $45^{+}$ions are thus not favored, as they are for ethylene glycol. Therefore, the proton affinity of the conjugate base of the $[\mathrm{M}+13]^{+}$product ion must be higher than that of neutral DME and considerably higher than that of the $[\mathrm{M}+13]^{+}$ion of ethylene glycol.

Reaction of ethylene glycol dimethyl ether. The reaction of ethylene glycol DME with the $45^{+}$ion (Figure 2c) follows a trend similar to that of the ethylene glycol reaction discussed previously. One difference in this case is that the $[\mathrm{M}+15]^{+}$product is formed instead of $[\mathrm{M}+13]^{+}$. Also, the $[\mathrm{M}+15]^{+}$product abundance does not fall off with increasing reaction time, and the $47^{+}$ion does not appear, even at long reaction times. Rather, a tiny abundance of methylated DME $61^{+}$ appears, presumably arising from methyl cation transfer between the $45^{+}$reagent ion and the neutral DME present in the system. The relative abundance of this peak is indeed so small that it is not distinguishable when plotted on the same scale as the other peaks. Also, the $[\mathrm{M}+15]^{+}$product ion does not have an acidic proton and would thus not be expected to transfer a proton to neutral DME.

Of particular note is the fact that the formation of $[\mathrm{M}-1]^{+}$and $[\mathrm{M}+1]^{+}$products predominate in this reaction profile. The presence of these two ions can be explained by the mechanism shown in Scheme II. The $[\mathrm{M}-1]^{+}$ion is presumably formed by hydride transfer from the ethylene glycol DME substrate to the $45^{+}$ cation, as described previously for the same process in the diol reaction. The resulting ion is then easily deprotonated by neutral ethylene glycol DME, and the reaction yields a neutral unsaturated ether and the $[\mathrm{M}+1]^{+}$ion. This proposed mechanism is substantiated by two observations.

At lower sample pressures, deprotonation of the $[\mathrm{M}-1]^{+}$ion by neutral analyte molecules, resulting in the formation of $[\mathrm{M}+1]^{+}$ions, is expected to be increasingly disfavored, because of the lower probability of collisions with the neutral ethylene glycol DME molecules. Indeed, the $[\mathrm{M}+1]^{+}$signal decreases dramatically as the sample pressure is lowered, whereas the $[\mathrm{M}-1]^{+}$signal remains high. Also, isolation of

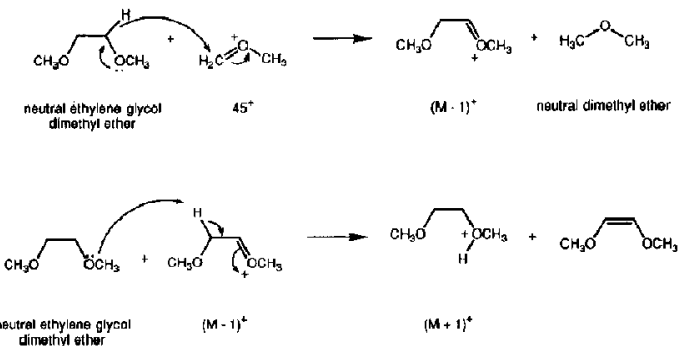

Scheme II. Formation of secondary products in the reactions of ethylene glycol DME with the $45^{+}$ion. 
the $[M-1]^{+}$ion, followed by long reaction times results in formation of $[M+1]^{+}$. This further supports the conclusion that the $[\mathrm{M}+1]^{+}$ion that appears in Figure $2 c$ is generated indirectly from reaction of $[M-$ $1]^{+}$, rather than as a direct product of the initial reaction of ethylene glycol DME with the $45^{+}$ion.

Finally, the rate of disappearance of the $45^{+}$ion is fastest for reaction with the diether and slowest for reaction with the diol. This is presumably due to the higher nucleophilicity of the substrates with increasing degree of oxygen atom substitution. The fact that the relative intensities of the final products of these reactions do not follow this trend indicates that there are two steps in each of the formation reactions. The first involves attack of the substrate on the $45^{+}$ion and its rate is dependent on the degree of attraction between the two reactants. The second is an intramolecular process that depends only on the structure and degree of functional group interaction within the system.

\section{Examination of Formation Mechanisms}

Formation of $[M+13]^{+}$. The fact that only the two compounds that possess hydroxyl groups form the $[\mathrm{M}+13]^{+}$product is in complete accord with the mechanism previously determined for this reaction in other systems $[9,12,24]$ and indicates that the same type of mechanism may be operative here. The proposed mechanism for the formation of $[\mathrm{M}+13]^{+}$ions from the substrates studied here is shown for ethylene glycol in Scheme III. Presumably, either of the two nucleophilic oxygen atoms of the ethylene glycol or ethylene glycol monomethy] ether substrates may attack the positively charged methylene carbon atom of the methoxymethylene cation $(\mathrm{m} / z$ 45) within the collision complexes previously described (Scheme $\mathrm{Ib}$ ), resulting in the covalently bound $[\mathrm{M}+45]^{+}$, adduct shown (Scheme $\mathrm{lb}$, upper path). Because of the higher nucleophilicity of the methoxyl group, the attack by the ether oxygen atom of ethylene glycol monomethyl ether may be slightly favored over hydroxyl oxygen atom attack; however, this small difference, with respect to the high internal energy available for promotion of the reaction, is not likely to cause a dramatic change in reactivity. The change is more likely manifested in the subsequent step of the reaction, which is clearly functional group dependent.

If a nearby acidic proton, such as the one remaining on an attacking hydroxyl oxygen atom, is available, it can be transferred to the oxygen atom of the DME portion of the adduct. An oxonium ion is thus formed, the carbon-oxygen bond is immediately cleaved, and a molecule of methanol is lost. The hydroxyl proton remaining on the unreacted functional group of the ethylene glycol adduct or the methoxyl-site adduct of ethylene glycol monomethyl ether is apparently not acidic enough or close enough to the receiving site to allow for efficient transfer. The transferred proton must therefore originate from the attacking functional group

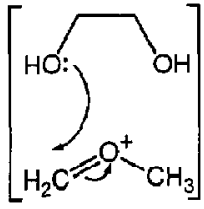

$(M+45)^{+}$

Collision Complex

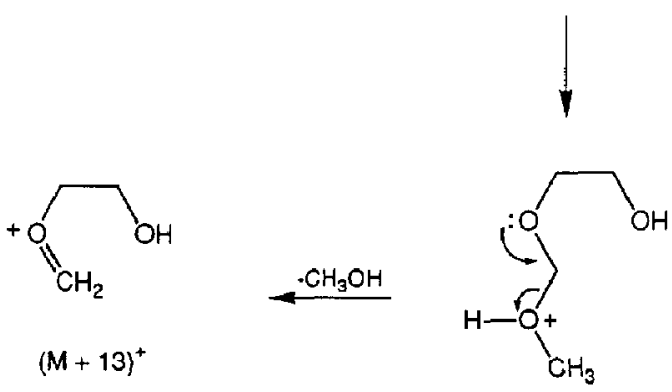

Scheme III. Representative formation mechanism for $[M+13]^{+}$ product ions.

for the fragmentation reaction to occur. It can thus be inferred that only hydroxyl-site $[M+45]^{+}$adducts of these molecules can give rise to the $[M+13]^{+}$product ion. Of the three substrates studied here, only ethylene glycol and its monomethyl ether are able to fulfill this requirement, and, accordingly, they are the only ones to exhibit formation of the $[\mathrm{M}+13]^{+}$product.

Formation of $[M+15]^{+}$. Thus, there remains the question of the fate of the monoether and diether methoxyl-site $[\mathrm{M}+45]^{+}$adducts that presumably form but are not directly observed. Because of the high internal energy of the collision complex and the inaccessibility of the $[M+13]^{+}$fragmentation pathway described before, an alternate deactivation route must be enabled. Two likely relaxation mechanisms other than the formation of $[M+13]^{+}$are dissociation to the starting reactants and fragmentation to form different products. Radiative relaxation is another possible cooling mechanism, but this process cannot be quantitated in the ion trap. Likewise, although dissociation of the adduct to reform the reactants is a likely occurrence, the extent of this dissociation cannot be measured because the collision complex is not directly isolable. A new product, $[M+15]^{+}$, is indeed observed for the reaction of ethylene glycol DME with the $45^{+}$ion of DME and indicates that alternate fragmentations are at least in part responsible for the disappearance of the $[\mathrm{M}+45]^{+}$adduct. Two mechanisms for this net transfer of a methyl cation from the $45^{+}$reagent ion to the neutral ethylene glycol DME can be envisaged. 


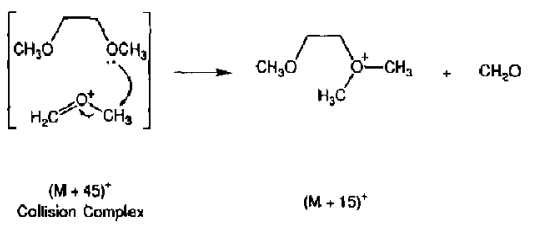

a
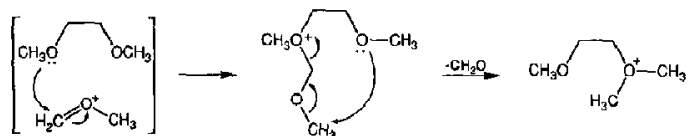

$(M+45)^{+}$ Collision Complex

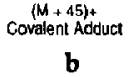$$
(M+95)^{+}
$$

Scheme IV. Proposed formation mechanisms for $[M+15]^{+}$ product ions: (a) displacement reaction; (b) rearrangement of the $[M+45]^{+}$adduct (see text).

The first of these is a simple displacement reaction, as illustrated in Scheme IVa [32]. Either of the methoxyl groups of the substrate may interact directly with the methyl group of the reagent ion within the collision complex. This interaction might then result in cleavage of the bond between the methyl group carbon atom and the oxygen atom of the reagent ion and the loss of neutral formaldehyde. Migration of the methyl cation to the substrate would thus occur without the intermediacy of an $[\mathrm{M}+45]^{+}$adduct. Examination of the resonance structures for the $45^{+}$ion indicates that a major portion of the positive charge on the $45^{+}$ion is expected to rest on the methylene carbon atom. The methyl carbon atom of the reagent ion would thus have to be sufficiently polarized to be attracted by the nucleophilic centers on the ethylene glycol DME substrate. If this is indeed the case, it is conceivable that the reaction proceeds by attack of the neutral substrate at this site; however, the absence of this product for the reactions of ethylene glycol and ethylene glycol monomethyl ether seems to belie this hypothesis.

An alternative mechanism involves a rearrangement of the $[M+45]^{+}$adduct, as shown in Scheme IVb. The unreacted functional group of the covalent $[\mathrm{M}+45]^{+}$ion attacks the end-methyl group, and its bond to the oxygen atom is displaced. Further electronic rearrangement gives loss of a molecule of formaldehyde and the $[M+15]^{+}$product ion. In this case, however, the methyl group is attached to the functional group that did not react with the $m / z 45$ cation. Although a similar mechanism could be invoked for the formation of $[M+15]^{+}$ions from ethylene glycol and ethylene glycol monomethyl ether adducts, this reaction is expected to be considerably less favored than the formation of $[M+13]^{+}$product ions for these species. Therefore, only substrates such as ethylene glycol DME that can give no alternative product are expected to undergo this process.

\section{Heats of Reaction}

To support the conclusions made in the present study, we have used semiempirical methods to estimate the thermochemistry of the processes in question. This type of analysis is especially useful in cases such as this, in which differences in potential reaction sites are to be considered. As demonstrated earlier, DME is a particularly selective reagent, and examination of the heats of reaction for the various processes that can occur gives insight into the reasons for this selectivity.

The estimated heats of formation for the $[\mathrm{M}+45]^{+}$ adducts of ethylene glycol, ethylene glycol monomethyl ether, and ethylene glycol DME and all of the possible $[\mathrm{M}+13]^{+}$and $[\mathbf{M}+15]^{+}$products are listed in Table 1. For ethylene glycol monomethyl ether, there are two entries included. The first concerns the ions resulting from reaction at the hydroxyl site, and the second concerns those resulting from reaction at the methoxyl site. Also listed are the approximate heats of reaction for the formation of the $[M+13]^{+}$and $[M+15]^{+}$ions by the mechanisms proposed. The values listed support the earlier conclusions concerning the observed reactive behavior of these compounds.

The activation energy required for the bond formation between the $45^{+}$ion and the diether portions of the collision complex serves to dissipate some of the excess internal energy of the complex; however, this process is not sufficient to completely cool the resulting adduct. The conditions of the ion trap mass spectrometer do not promote complete collisional deactivation of the newly formed ions. Thus, the covalently bound $[\mathrm{M}+45]^{+}$product spontaneously dissociates in one of the ways previously discussed. The low heats of reaction are thus consistent with the fact that no $[\mathrm{M}+45]^{+}$ions are observed for these substrates in the ion trap mass spectrometer and support the idea that the $[M+13]^{+}$and $[M+15]^{+}$ions observed are the secondary products of the ion-molecule reactions between the glycols and the $45^{+}$ion.

In Table 1, the values shown for the two different products for ethylene glycol monomethyl ether give further insight into the nature of the $[\mathrm{M}+45]^{+}$formation reaction. The hydroxyl-site $[\mathrm{M}+45]^{+}$adduct of ethylene glycol monomethyl ether has a heat of formation very similar to that of the ethylene glycol adduct (Table 1). As expected, the heats of formation for the two possible methoxyl-site adducts (ethylene glycol monomethyl ether and ethylene glycol DME, Table 1) are likewise similar. According to these estimated values, under thermodynamic control, the hydroxyl-site adduct would be heavily favored over the methoxylsite isomer; however, because of the high internal energy of the ion and its immediate dissociation, there is no indication that this formation reaction indeed has any effect on the outcome of the overall ion-molecule reaction. It is significant, however, that the $[M+45]^{+}$ formation reaction at the two different sites for the same substrate is significantly less exothermic for reac- 
Table 1. Estimated heats of formation for possible reaction products and intermediate products

\begin{tabular}{|c|c|c|c|c|c|c|}
\hline \multirow[b]{2}{*}{ Compound } & \multirow[b]{2}{*}{ Structure } & \multicolumn{3}{|c|}{ Heat of formation (kcal /mol) } & \multicolumn{2}{|c|}{ Heat of Reaction (kcal/mol) } \\
\hline & & {$[\mathrm{M}+45]^{+}$} & {$[M+13]^{+}$} & {$[\mathbf{M}+15]^{+}$} & {$[\mathrm{M}+13]^{+}$} & {$[M+15]^{+}$} \\
\hline Ethylene glycol & но & 21 & 94 & 79 & -1 & 6 \\
\hline \multicolumn{7}{|l|}{ Ethylene glycol } \\
\hline monomethyleth & & & & & & \\
\hline Hydroxyl site & & 24 & 99 & 80 & -3 & 0 \\
\hline Methoxyl site & & 35 & c & 83 & c & 3 \\
\hline \multicolumn{7}{|l|}{ Ethylene glycol } \\
\hline dimethyl ether & $\mathrm{CH}_{2} \mathrm{O}^{\prime}$ & 34 & c & 76 & c & -8 \\
\hline
\end{tabular}

tion at the methoxyl site. There is thus less excess internal energy in the adduct after the reaction, and the likelihood that the adduct will undergo a high-energy fragmentation route is considerably smaller for the methoxyl-site isomer.

The heats of formation for the $[M+15]^{+}$product ions are lower than those for the $[\mathrm{M}+13]^{+}$ions, but the $[\mathrm{M}+13]^{+}$formation reaction is favored over that for $[M+15]^{+}$, even when both products can form (ethylene glycol monomethyl ether, Table 1). This occurrence is readily explained by examination of the overall heats of reaction for the two processes. The heats of reaction for the $[M+13]^{+}$formation reaction are substantially different from those for formation of $[\mathrm{M}+15]^{+}$. Whereas the $[M+13]^{+}$reaction is very slightly exothermic, the $[\mathrm{M}+15]^{+}$reaction for the same compound is slightly endothermic. Thus, it is not surprising that for ethylene glycol, only the $[M+13]^{+}$ ion is formed. On the other hand, the only possible product for ethylene glycol DME is $[M+15]^{+}$, and for this substrate, the reaction is exothermic. It is therefore not surprising that exclusive formation of $[\mathrm{M}+15]^{+}$is observed.

For ethylene glycol monomethyl ether, both the methyl cation transfer and methylene substitution processes are possible; however, by comparing the heats of reaction for the two paths, it is noted that whereas the $[\mathrm{M}+15]^{+}$formation reaction is disfavored for both reaction sites, $[M+13]^{+}$formation at the hydroxyl site is slightly exothermic. Again, these data support the assertion that the $[M+13]^{+}$pathway is the favored dissociation route for the $[M \cdot 145]^{+}$adducts.

\section{Conclusions}

The ion of $m / z 45$ from DME is a selective reagent that can unambiguously distinguish between hydroxyl and methoxyl groups. Using this reagent, we have demonstrated that the natures of various functional groups within molecules can dramatically affect the mechanisms and outcomes of reactions. This offers great future opportunities for the development of siteselective reagents for use in both simple organic systems and more complex biochemical molecules.

\section{Acknowledgments}

This work was supported by grants from the Welch Foundation (F-1155), the Petroleum Research Fund (22270-G5), the National Science Foundation (CHE-9122699), and the National Institutes of Health (R01-GM46723-01). Generous assistance with the computer calculations from Professor John E. Bartmess is also gratefully acknowledged.

\section{References}

1. Hughes, E. D. Q. Rev. Chem. Soc. 1948, 2, 107.

2. Brown, H. C.; Fletcher, R. S. J. Am. Chem. Soc. 1949, 71, 1845.

3. Perez, I. D.; Phagouape, L. M.; Davico, G. E. I. Phys. Org. Chem. 1989, 2, 225.

4. Wolf, R.; Grutzmacher, H. F. New J. Chem. 1990, 14, 379.

5. Mishima, M.; Inoue, H.; Fujio, M.; Tsuno, Y. Tetrachedron Lett. $1990,31,685$.

6. Manion, J. A.; Louw, R. I. Chem. Soc. Perkin Trans. 2 1990, 551.

7. Hrusak, J.; Tkaczyk, M. Org. Mass Spectrom. 1990, 25, 214.

8. Jerkovich, G.; Hankovszky, H. O.; Hideg, K. Org. Mass Spectrom. 1990, 25, 67.

9. Eichmann, E. S.; Alvarez, E.; Brodbelt, J. S. J. Am. Soc. Mass Spectrom. 1992, 3, 535.

10. Harrison, A. G.; Kallury, R. K. M. R. Org. Mass Spectrom. $1980,15,277$.

11. Fales, H. M.; Milne, G. W. A.; Nicholson, R. S. Anal. Chem. 1971, 43, 1785.

12. Eichmann, E. S; Brodbelt, J. S. I. Am. Soc. Mass Spectrom., in press.

13. Liou, C.C.; Eichmann, E. S.; Brodbelt, J. S. Org. Mass Spectrom. 1992, 27, 1098.

14. Morton, T. H.; Beauchamp. J. L. J. Am. Chem. Soc. 1975, 97 , 2355.

15. Fenselau, C. C.; Robinson, C. H. J. Am. Chem. Soc. 1971, 93, 3070 .

16. Aue, D. H.; Webb, H. M.; Bowers, M. T. J. Am. Chem. Soc. $1976,98,311$.

17. Arnett, E. M.; Chawla, B.; Bell, L.; Taagepera, M.; Hehre, W. J.; Taft R. W. I. Am. Chem. Soc. 1977, $99,5729$.

18. Rawn, J. D. Biachemistry; Harper and Row: New York, 1983. 
19. Busch, K. L.; Glish, G. L.; McLuckey, S. A. Mass Spectrometry/Mass Spectrometry: Techniques and Applications of Tandem Mass Spectrometry; VCH: New York, 1988.

20. Mabud, M. D. A; Dekrey, M. J.; Cooks, R. G. Int. I. Mass Spectrom. Ion Processes 1985, 67, 285.

21. Munson, M. S. B.; Field, F. H.; I. Am. Chem. Soc. 1966, 88, 2621.

22. Harrison, A. G. Chemical Ionization Mass Spectrometry; CRC: Boca Raton, FL, 1983.

23. Vairamani, M.; Ali Mirza, U.; Srivinas, R. Mass Spectrom. Rev. 1990, 9, 235.

24. Brodbelt, J.; Liou, C.-C. Donovan, T. Anal. Chem. 1991, 63, 1205.

25. Angelini, G.; Sparapani, C.; Speranza, M. J. Am. Chem. Soc. $1992,112,3060$.
26. Robin, D.; Hopilliard, Y;; Audier, H. Org. Mass Spectrom. $1988,23,370$.

27. Wood, K. V.; Burinsky, D. J.; Cameron, D.; Cooks, R. G. J. Org. Chem. 1983, 48, 5236.

28. Louris, J. N.; Cooks, R. G.; Syka, J. E. P.; Kelley, P. E.; Stafford, G. C. Jr; Todd, J. F. J. Anal. Chem. 1987, 159, 1677.

29. Louris, J. N.; Brodbelt, J. S.; Cooks, R. G.; Glish, G. L.; Vari Berkel, G. J.; McLuckey, S. A. Int. I. Mass Spectrom. Ion Processes 1990, 96, 117.

30. Weber-Grabau, M.; Kelley, P. E.; Syka, J. E. P.; Bradshaw, S. C.; Brodbelt, J. B. Proceedings of the 35th AMS Conference on Mass Spectrometry and Allied Topics; Denver, CO, 1987; $\mathrm{p} 1114$.

31. Donovan, T.; Brodbelt, J. Biol. Mass Spectrom. 1991, 21, 254.

32. Van Doorn, R.; Nibbering, N. M. M. Org. Mass Spectrom. $1978,13,527$. 